\title{
ATLAS Construction Status
}

\author{
P. Jenni \\ CERN, Geneva, Switzerland
}

Received on 20 January, 2005

\begin{abstract}
The ATLAS detector is being constructed at the LHC, in view of a data-taking startup in 2007. This report concentrates on the progress and the technical challenges of the detector construction, and summarizes the status of the work as of August 2004. The project is on track to allow the highly motivated ATLAS Collaboration to enter into a new exploratory domain of high-energy physics in 2007.
\end{abstract}

Keywords: ATLAS detector; Large Hadron Collider (LHC)

\section{INTRODUCTION}

The ATLAS Collaboration is constructing a general purpose detector to exploit the rich discovery potential of new physics which will be offered by the Large Hadron Collider (LHC) at the European Laboratory for Particle Physics (CERN) located near Geneva, Switzerland. The LHC construction is well under way for starting up operation with proton-proton interactions at $14 \mathrm{TeV}$ collision energy in summer 2007.

Besides the construction and the integration of detector components for the general-purpose ATLAS detector, which have steadily progressed over the past years, the installation in the huge underground cavern $(100 \mathrm{~m}$ below ground, about $50 \mathrm{~m}$ long with a diameter of about $35 \mathrm{~m}$ ) has now entered into the focus work. Since the delivery of the cavern in June 2003, the first phase of the installation - the infrastructure and main supports - has almost been completed. The first active detector components (the Tile Calorimeter modules) have been lowered into the cavern on March 1st, 2004. The construction of detector components is more than $70 \%$ complete.

The ATLAS Collaboration currently consists of 151 institutions from 34 countries, counting approximately 1700 scientific authors. The team from the Universidade Federal do Rio de Janeiro (COPPE/EE/IF) has been involved in the conception and development of the detector concept since the beginning, starting with active participation in the calorimeter R\&D in 1998. Since then the team has contributed in an excellent and visible way to all phases of the detector construction, electronics and software developments, and the physics studies in order to optimize the layout and assess the discovery potential of the experiment.

The ATLAS detector concept is based on a superconducting magnet system with a central solenoid around the Inner Detector and large air-core toroid magnets for the Muon Spectrometer with, between the two, the Liquid Argon (LAr) and Tile Calorimeters. A hierarchical Trigger and Data Acquisition System (DAQ) will provide the data for the collaborationwide computing and physics analysis activities.

\section{MAGNET SYSTEM}

The ATLAS superconducting magnet system consists of a central solenoid, a barrel, and two end cap toroids. They are serviced by common cryogenics, power, and control systems, for which most components have already been delivered and installed in the experimental cavern. The Helium refrigerators and cold boxes have been lowered into the service cavern and are being commissioned. The central solenoid has been successfully inserted and integrated into the common cryostat with the LAr barrel electromagnetic calorimeter end of February 2004. Functional cold tests of the solenoid have been performed in the period June-July 2004 on the surface. The solenoid was energized to its maximal current, plus a margin of $8 \%$. Several induced quenches have proven the robustness of this device. The next step consists in the lowering into the cavern of the solenoid within the LAr cryostat, which is foreseen for October 2004.

The barrel toroids (BT) are all under assembly at CERN. The cold mass integration for the eight coils has been completed in October 2003, and they have now been equipped with Helium cooling lines and all services. All eight vacuum vessels are completed as well, and ready to receive the cold masses. However, the thermal shield delivery and installation have suffered from important delays (about ten months), first due to various design issues and, since more than half a year, due to serious industrial production problems, which were solved beginning of 2004. The thermal shield panels were installed on the first 4 coils (August 2004). By now the installation of the Nitrogen cooling lines has become a routine operation. The first two coils are also being equipped with super-insulation, and the first coil "cryostating" was performed in June 2004. This will lead to the first cold operational test in August, and readiness for installation at the end of September 2004. The last coil completion is scheduled for May 2005. In the mean time the preparations for the warm structure, the cryogenic feed-ring connecting all eight colis, and all the installation tooling have progressed sufficiently fast to meet the late availability of the coils themselves.

The construction of the two end cap toroids (ECT) follows a different path. The two vacuum vessels are ready at CERN and equipped as far as possible with super-insulation and heat shields. The coil winding and cold-mass assembly are proceeding well at the factory, with the exception of the cool- 


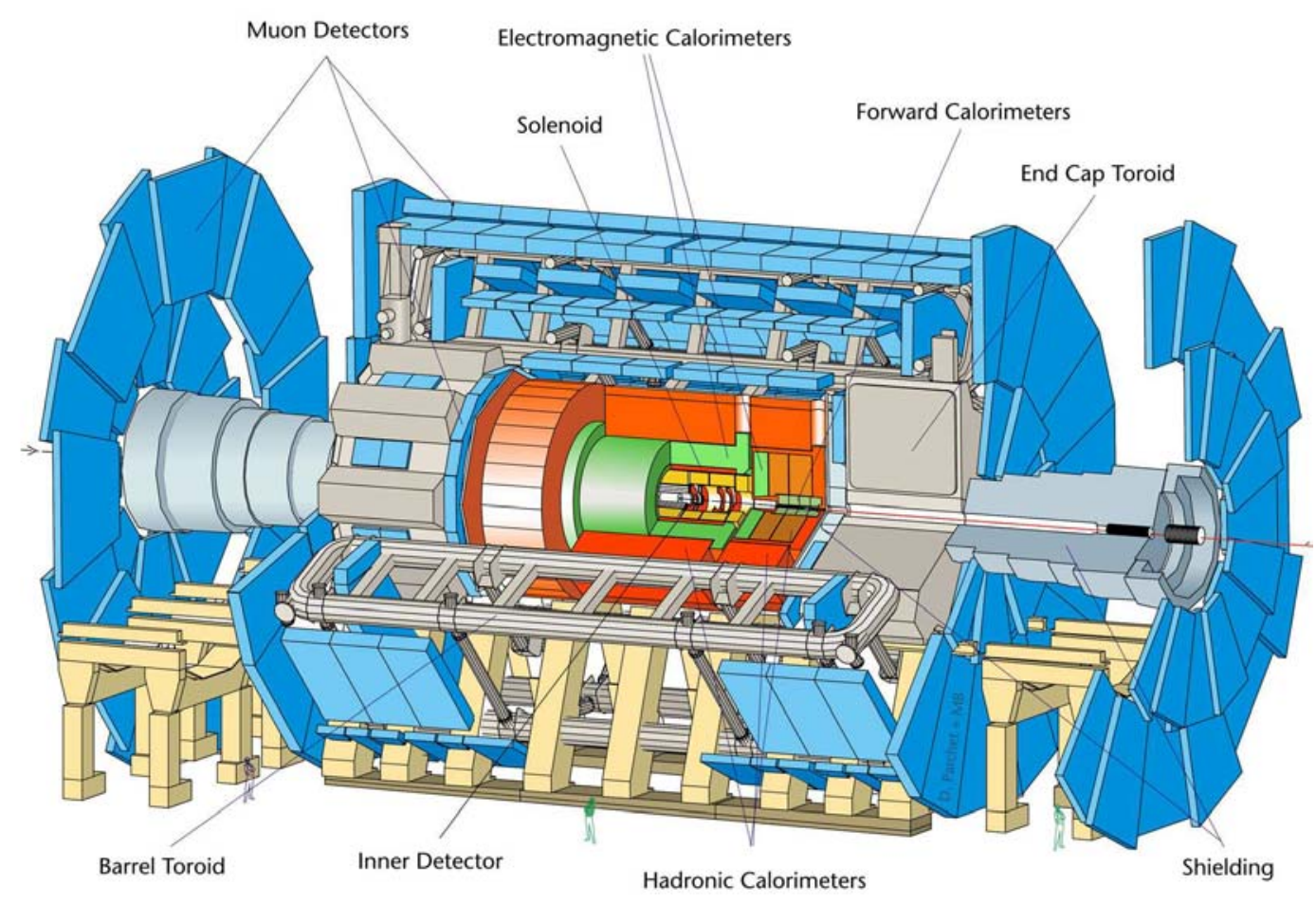

FIG. 1: Lay-out of the ATLAS detector with its major sub-system components. The diameter is about $25 \mathrm{~m}$, the total length about $46 \mathrm{~m}$, and the weight 7000 Tons.

ing line production. All 16 coils have been wound, and the eight ones for the first ECT have been successfully impregnated. The project had to be reorganized in November 2003, by moving the cooling lines out of the scope of the contract, and adding it to the integration work at CERN. All components of the cold mass for the first ECT have arrived at CERN for integration in Summer 2004, those for the second one are expected for January 2005. The first ECT will be assembled by the beginning of 2005 .

\section{MUON SPECTROMETER}

The ATLAS Muon Spectrometer is instrumented with precision chambers for momentum measurement, complemented with fast chambers for triggering. Several thousands of chambers have to be constructed. All chamber subsystems, optical alignment components and supports are well advanced in the series construction. System aspects, such as the crucial alignment monitoring and the calibration, were studied in a largescale test beam set up at the CERN SPS with production line detectors.

\section{A. Precision Chambers}

The 13 production sites for the Monitored Drift Tube (MDT) chambers are slightly ahead of the planned construction schedule for the bare chambers, with about $88 \%$ of them assembled. About $60 \%$ of the MDTs for the initial detector are already integrated with their services. The MDT electronics series production is in progress, and the mounting on the chambers has started in April 2004. Cathode Strip Chambers (CSCs) are used in the innermost end cap region because of the high radiation fluxes. All these chambers have been produced.

\section{B. Trigger Chambers}

The barrel region is equipped with Resistive Plate Chambers (RPC). After a successful effort to improve the Quality Control steps, production is now proceeding at full-speed with about $80 \%$ of the gas volumes delivered, and $60 \%$ of the chamber modules assembled. The production rate has reached the level required for construction completion as scheduled, for end of 2004. A sample of early series chambers is be- 


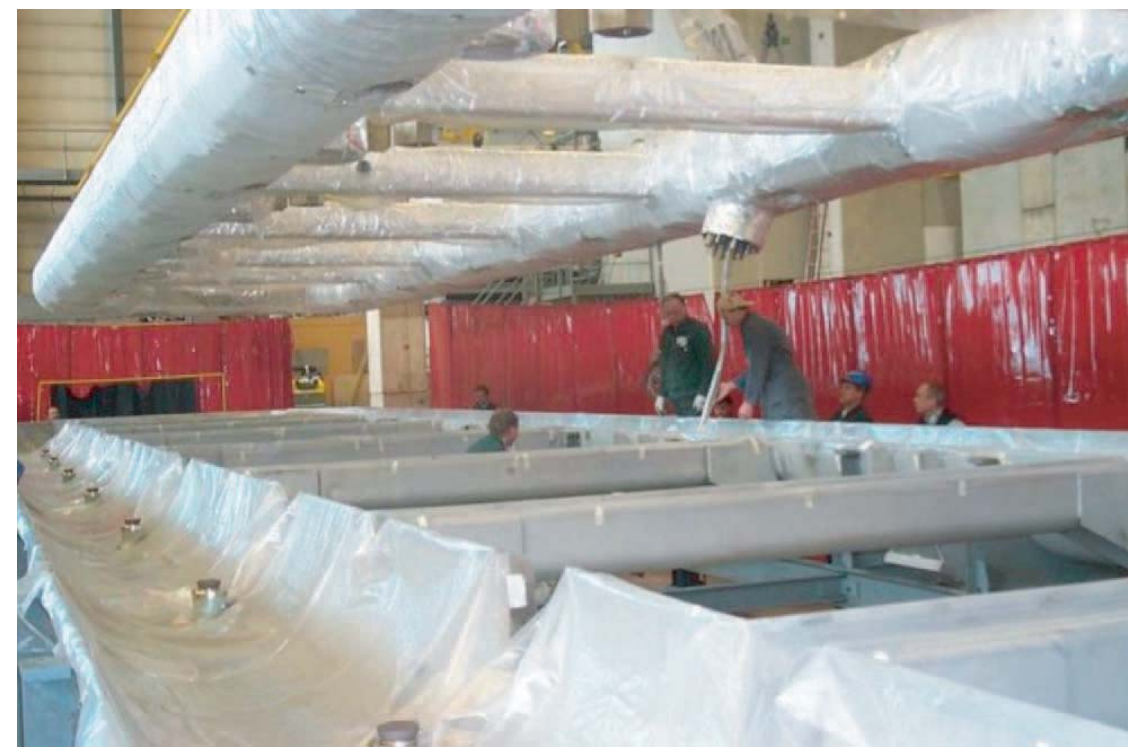

FIG. 2: Insertion of the second BT coil in its cryostat. The coil cold mass and thermal shielding are inside the multilayered insulation system wrapped around them.

ing subjected since end 2002 to final radiation qualification tests in the dedicated X5 beam irradiation facility at the CERN SPS, and is showing good results throughout. The end cap regions are instrumented with Thin Gap Chambers (TGC) that are able to cope with high rates. The TGC series fabrication is well advanced, with more than $87 \%$ of all chambers assembled, and $50 \%$ of them fully tested. More than $1 / 3$ of all chambers have arrived to CERN. The trigger chamber electronics was validated in a system test using an LHC-like beam structure in the SPS beam, after an initial run which uncovered some, by now well understood and corrected, problems.

\section{Muon Spectrometer System Aspects}

The large-scale test facility for alignment, mechanical, and many other system aspects at the SPS H8 beam is fully operational with sample series chamber stations. The design work for the large end cap chamber support structures ("Big Wheels") has been finalized after further design iterations to improve stability. Their procurement has now been launched, albeit with more delays than anticipated. The pre-assembly and testing of the precision chamber stations (1 MDT +2 RPCs) has just started. Large batches of chambers are arriving regularly at CERN.

\section{THE CALORIMETERS}

All the sub-systems of the Calorimeters have finished their module construction phase. The activities have shifted to the assembly and integration phase, and to the detailed planning for the installation and commissioning. A large-scale test beam campaign has started during 2004 with the aim to reproduce and study a wedge of the combined calorimeter system $(\mathrm{EM}+\mathrm{HAD})$.

\section{A. LAr Calorimeters}

Both barrel cylinders have been assembled and successfully inserted into the cryostat. Initial electrical tests show very good results. For the end cap electromagnetic (EM) calorimeter the modules construction was completed as scheduled in February 2004, including restacking of an early module which was slightly failing the acceptance criteria. The first of the two end cap EM wheels has been assembled, tested, inserted successfully into the cryostat, and cabled, following the presampler installation. Very good warm test results have been achieved. The second wheel assembly was finished end of April, followed by the insertion into its cryostat in May 2004.

The HEC (LAr Hadronic End Cap) series production is complete, with all modules stacked and cold-tested. These have been assembled into four wheels, two on each side. The insertion into the first end cap cryostat has been successful, including commissioning tests at room temperature. The cold test will follow in October 2004. The insertion into the second cryostat has to await the EM calorimeter readiness after testing.

The SPS calibration run for the first complete forward calorimeter (FCAL) ended successfully. The insertion of the FCAL into the first end cap cryostat is scheduled for August 2004. The assembly of the modules for the second side is also complete, all cold-tests have been done.

After the insertion of the EM calorimeters and the solenoid, the barrel cryostat has been prepared for the final cold tests at 
the surface. The tests progress well and will end in August 2004, before the installation in the cavern in October 2004. In June, the barrel EM calorimeter, inside its cryostat, has been cooled down and filled with 53 tons of liquid Argon. A slower cool-down with gas (instead of liquid) Nitrogen has been used for the barrel in order to minimize mechanical risks. A modification of the cooling procedure for the end caps is under evaluation. Also because the final welding of the barrel cold covers has been completed successfully, after various tests, it has been decided to weld the covers (cold flanges) of both end caps as well. The first of the two end cap cryostats now containing the pre-sampler, the pre-assembled EM and HEC wheels, is ready for this final closing and welding.

Very good progress was made on the cryogenics plant, with major components installed. The two large LAr dewars are in place in the underground cavern; a third dewar for the Nitrogen cooling, which was delayed for technical reasons, has now been installed, with a special and unconventional procedure.

Final prototypes of both, front-end (FE) and back-end, electronics have been tested successfully. The digital chips in DMILL technology were finally delivered after some critical delays and manufacturing risks. The first radiation tolerant FE boards were produced and operated already in early 2003, as were other, radiation-tolerant, boards for calibrations and controls, including radiation tolerant voltage regulators. The FE board mass production has started. The back-end electronics, which consists mainly of the Read Out Driver (ROD) system, is also progressing, with prototypes and design reviews for several components passed. Detailed installation and commissioning plans for the electronics have been prepared.

\section{B. The Tile Calorimeter}

The Brazilian collaborators from UFRJ have been involved very actively in this sub-system project, since the early $R \& D$ phase, starting in 1988. Componets for the trigger and readout electronics have been developed at UFRJ, and built in Brazilian industry.

The module construction and instrumentation with optical components have been completed since early 2003. The preassembly, and disassembly for transport, of the first extended barrel cylinder as well as of the barrel cylinder have been successfully completed on the surface.

A major milestone was passed with a load test during the barrel disassembly, applying a weight corresponding to the one of the full LAr barrel calorimeter, solenoid and ID detector systems. The Tile Calorimeter electronics components are in fabrication, and their assembly into the "drawer" system housing all on-detector electronics circuits is now progressing well, on an accelerated pace. The Tile Calorimeter community has managed to anticipate the installation in the underground cavern, by successfully starting on 1st March with the lowering of the ensemble of the bottom eight barrel modules (as limited by the crane capacity). Since then, assembly proceeds steadily. Half of the barrel is assembled and is waiting for the LAr cryostat, before one proceeds with the second half.

\section{INNER DETECTORS}

The Inner Detector (ID) combines three concentric subsystem layers, namely (from inside out), the pixel detectors, the silicon detectors and the transition radiation straw tracker. The component fabrication is advanced for all of them, and in some cases completed. The module production has entered series construction. An important effort is the integration of the three subsystems, and their common ID infrastructure and services, for which preparations have progressed further according to schedule.

\section{A. Pixel Detector}

The pixel sensor series fabrication has been launched and is now at the $60 \%$ completion level. The FE electronics in radiation hard deep sub-micron (DSM) technology is in full series production. Pre-series prototype modules with final services were irradiated and tested successfully. Bump-bonding orders have been placed with a few manufacturers which have each produced about 30 modules. All high-tech light-weight mechanics support parts are well advanced and production clusters have started assembly of first production-quality modules onto staves and disks. System tests with realistic services have demonstrated design performance.

\section{B. Silicon Detector (SCT)}

The SCT sensor fabrication and acceptance testing is complete. The FE electronics fabrication is finished as well. The barrel module construction is progressing at the four sites, and more than $80 \%$ of the modules have been produced, even though delays have occurred because of temporary hybrid and pitch adaptor problems. The barrel support structures, C-fibre cylinders, have been delivered and much work is now concentrated on fitting them with services.

Technical challenges that have been recently overcome, include the delicate harness structures (thin service cable assemblies) and the low-material cooling lines. The complex assembly of modules on the barrel structures is on the critical path. Assembly of the first cylinder is planned for September 2004. The end cap module production had a slow start-up for various technical issues with components over the past years, but the major assembly sites are now qualified and produce good modules. The critical delivery of hybrids is now also overcome. The support structures for the end cap SCT advance well in fabrication and the services assembly has started as well, with the first disk being instrumented. This part of ATLAS is on the critical path, point of view schedule. The design and production of the off-detector read-out electronics and the power supplies are progressing well. 


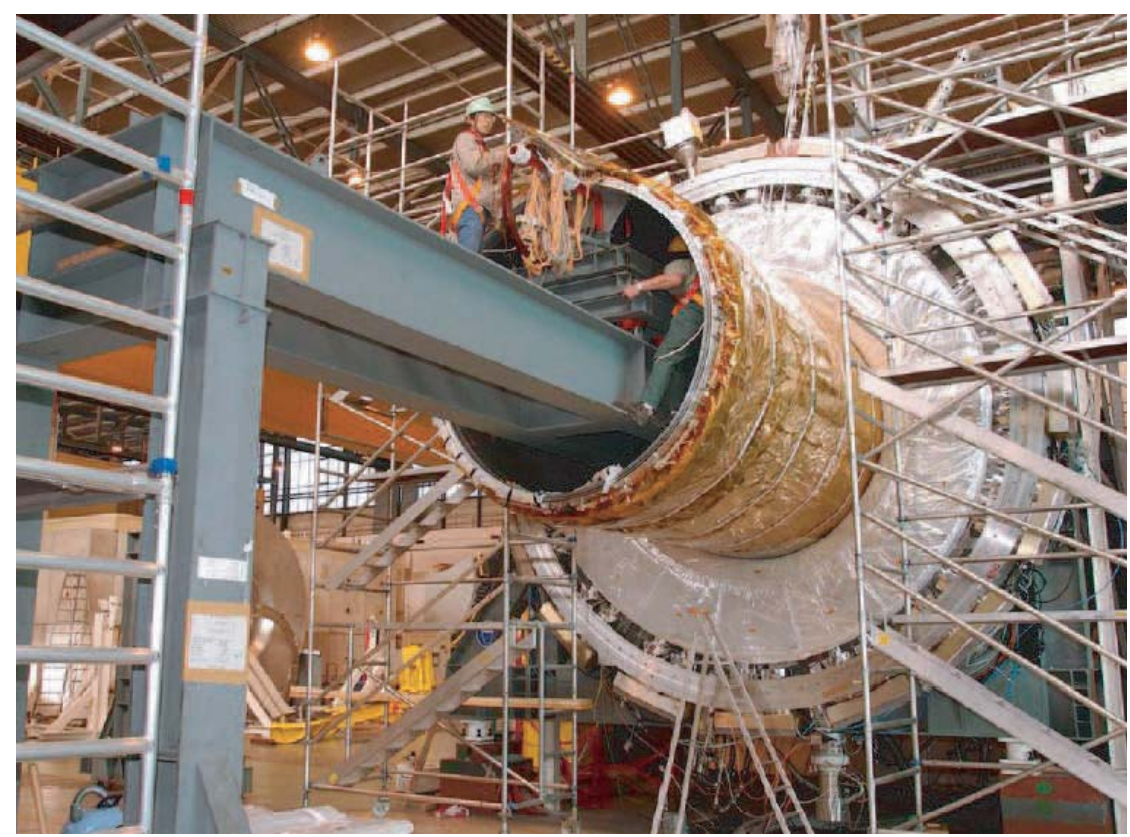

FIG. 3: LAr barrel calorimeter fully assembled. The central solenoid is being inserted into the common cryostat. In July 2004 this system has been cooled down and the solenoid was energized to its full current, including an $8 \%$ safety margin.
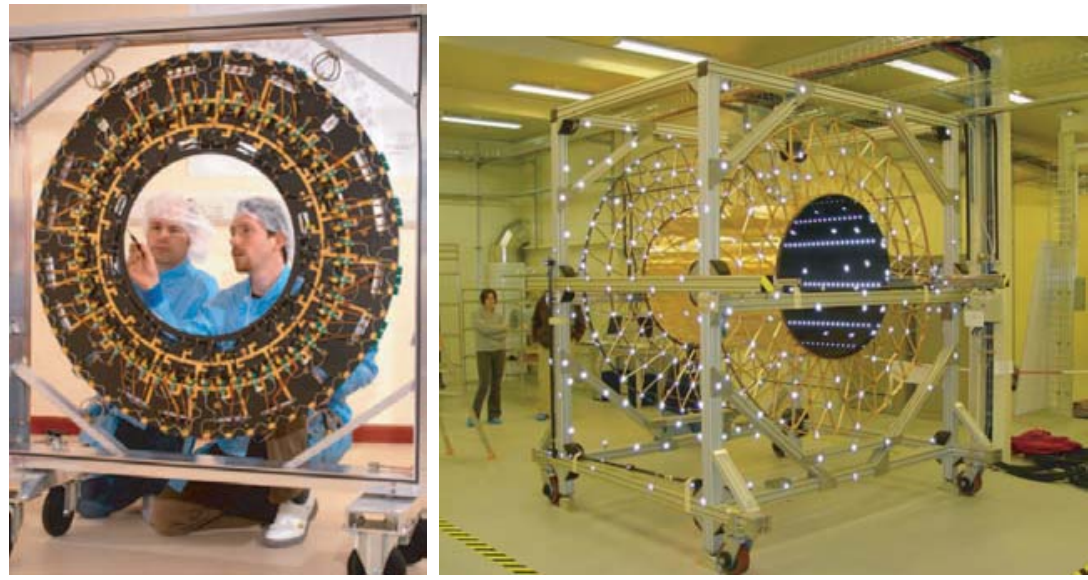

FIG. 4: First SCT forward disk being instrumented with all services. On the right, the barrel TRT support structure, just before insertion of the first layer of modules.

\section{Transition Radiation Tracker (TRT)}

The mechanical construction and wire stringing of the barrel TRT modules is completed, and the gain mapping is far advanced. All barrel modules are at CERN, being prepared for integration into the barrel support structure, delivered as well. The end cap wheel assembly is proceeding at the two sites, finally with the anticipated production rate after the delamination problems with the most critical component, the socalled web-circuits, have been solved. However, the schedule for the second end cap remains critical due to the accumulated delay. The FE electronics chips production has been successfully completed, including the one in DMILL technology. The first layer of barrel modules have been installed inside their final support structure in a large clean room integration facility (SR1) constructed for the entire ID and now operational near the surface building at the ATLAS detector site. 


\section{TRIGGER AND DATA ACQUISITION}

ATLAS uses multi-level triggers that provide rapid rejection of high-rate backgrounds to reduce the data output to mass storage down to an affordable rate. The fast, first-level trigger (custom electronics) is based on calorimetry and muon spectrometer information. At higher levels, information from other subdetectors (like the Inner Detector e.g.) provides decisions through especially devised algorithms using, at full granularity, a fraction of each entire event. At the highest level, the selection is refined with the capability of full event reconstruction. The UFRJ group with its electronics and computing expert knowledge is involved in various aspects of this part of the ATLAS project.

\section{A. Level-1 Trigger}

The level-1 Trigger system activities (with the sub-systems calorimeter, muon and central trigger logics) have progressed in the final ASICs developments and in testing fullfunctionality prototype modules. Major efforts have been spent on extended system tests ("slice-tests") of the detector and electronics to validate and improve the final designs and to be ready for the series fabrication start-up for most components. The muon trigger sub-system, both for the barrel and the end caps, operated successfully in a $25 \mathrm{~ns}$ bunch-spaced beam test validating the electronics chains. The final version of all parts of the on-detector system is on schedule for verification in the 2004 test beam runs. The delivery of the final trigger electronics to be mounted on the muon chambers before final installation is now on the critical path. Although there have been some delays for the Central Trigger Processor subsystem, it will be available on schedule for installation; design improvements allow increased trigger combination flexibility in the physics selection.

\section{B. High Level Trigger (HLT), DAQ and Detector Control System (DCS)}

A major milestone was reached with the recommendation for approval by the LHCC, the CERN committee reviewing the detector construction, of the Technical Design Report (TDR) for the HLT, DAQ and Controls at the end of 2003. The TDR is based on an extensive large-scale prototype system realized and tested over the past years. The final architectural choices for the Read-Out System (ROS) have been made in a timely manner. The work can now proceed in a well-defined framework, which still leaves enough flexibility to benefit from future developments in information technology to optimize the deployment of some components of the data flow. In these considerations the scalability of the HLT/DAQ system is particularly important for staging needs during the initial running of ATLAS. The future HLT/DAQ developments will be validated further in complete laboratory "test-beds" and in a real application for the large combined test beam runs during 2004. The DCS design, with its general purpose Embedded Local Monitor Board (ELMB) and the LHC-wide common supervisor system (SCADA), continued to be field-proven in many test beam and QC applications. All interfaces with the detector systems on one hand, and DAQ on the other, have been defined and are in development.

\section{UNDERGROUND INSTALLATION}

Since the experimental cavern hand-over to the Collaboration end of June 2003, very major installation progress can be reported. The installation proceeds along a detailed planning with six phases, comprising more than 2000 work packages. The first-phase installation (infrastructures) in the main cavern UX15 as well as in the large service cavern USA15 has been completed end of January 2004, with the exception of the delayed cooling and ventilation duct installations that are just being finished. Main elements installed are the cranes, metallic structures, external cryogenics, electrical services, survey network, gas piping, racks and cable trays. The second-phase installation has started with major elements: the complete main detector support structure (bed-plates, feet and rails) is in place and surveyed.

The first of the moveable support structures at the end of the detector, the so-called trucks, is in place and, most importantly, the assembly of the Tile Calorimeter barrel in situ has started on March 1st, 2004. This activity has been anticipated by more than two months because of the late availability of the first BT coil, for which at least the installation preparation has just started with the placement of the dedicated temporary mounting platforms. The plan is to be ready to lower the first BT coil into the cavern in October 2004. In the mean time, the lower half of the Tile Calorimeter barrel has been completed, ready to receive the complete LAr barrel cryostat. The planning of the commissioning is ongoing, spanning from component testing after installation to global cosmic ray data taking end of 2006, followed by using LHC beams as soon as they will become available in 2007 .

\section{COMPUTING, SOFTWARE AND PHYSICS PREPARATIONS}

ATLAS is currently engaged in the massive Data Challenge 2 (DC2) computing campaign, which is a test at the $10 \%$ level of the data rate expected at LHC start-up. The data is simulated with GEANT4 and the DC2 operation is based on POOL persistency and GRID infrastructure. The UFRJ group contributes actively to the computing infrastructure developments, especially by Web tools that help efficient data and information management in the worldwide Collaboration.

The goal of the DCs is to gain experience with worldwide distributed production and analysis environments. GRID middleware and tools are used (jointly for the three used GRID flavours LCG-2, NORDUGRID and GRID-3). The data will be used for large-scale physics studies, and also to test and shape the so-called ATLAS computing model, which will be 


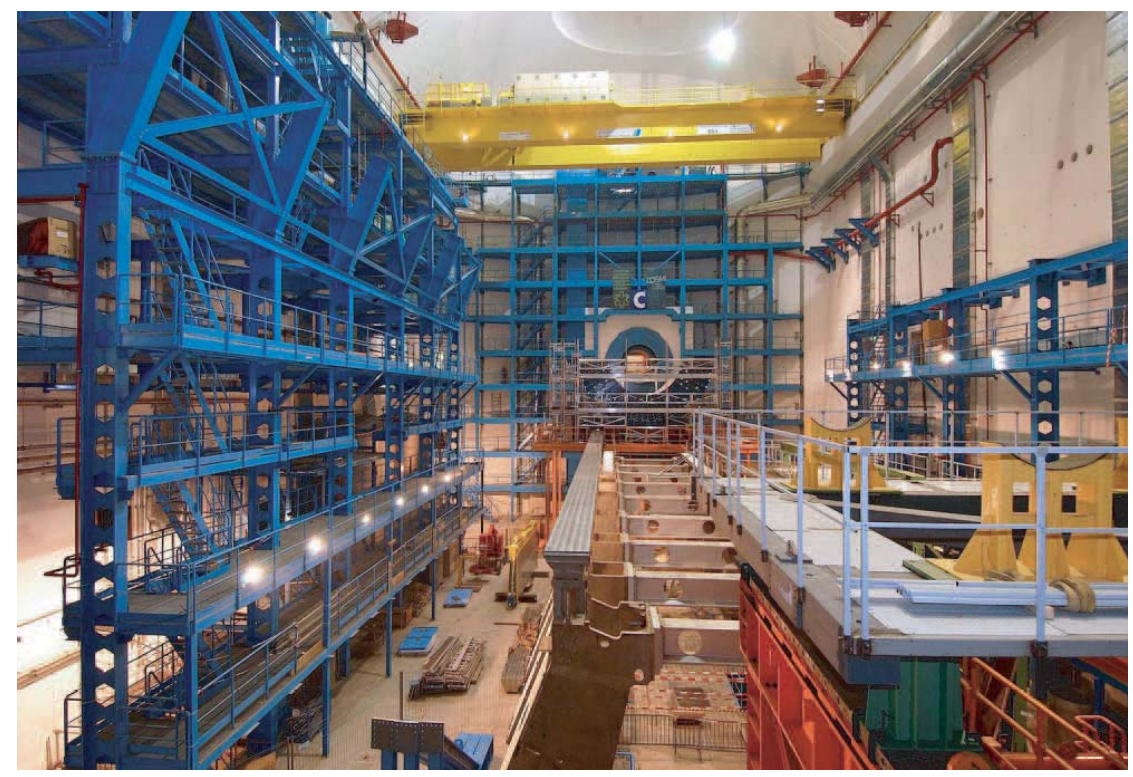

FIG. 5: ATLAS underground experimental cavern, status May 2004. Visible is the half cylinder of the barrel Tile Calorimeter, which has been assembled underground since March 2004.

a major input to the estimates for the future resources requirements. Even though DC2 is progressing steadily, the largescale operation is also revealing several areas where improvements need to be achieved in order to reach the planned efficient and smooth running of the collaboration-wide computing for LHC turn-on. The DC2 is embedded into the framework of the CERN LHC Computing Grid Project (LCG). In fact ATLAS is a very active partner in the LCG.

The exciting physics potential of the ATLAS experiment at LHC has been amply documented in the literature and the Physics and Detector Performance Technical Design Reports, available on the Web. The detector design has been optimised to cover the largest possible range of LHC physics: searches for Higgs Bosons and alternative schemes for the spontaneous symmetry-breaking mechanism; searches for Supersymmetric particles (SUSY), new Gauge Bosons, Leptoquarks, and Quark and Lepton Compositeness indicating extensions to the Standard Model and New Physics beyond it (for exam- ple extra dimensions); studies of the origin of $\mathrm{CP}$ violation via high-precision measurements of $\mathrm{CP}$-violating $\mathrm{B}$-decays; high-precision measurements of the third quark family such as the Top Quark mass and decay properties, rare decays of B-hadrons, spectroscopy of rare B-hadrons, and B0-mixing. It can be noted that many of these fundamental investigations have very direct links to Cosmology and Astroparticle Physics, which will be exploited to the mutual benefit of both fields.

\section{ACKNOWLEDGEMENTS}

It is a great pleasure to acknowledge the friendly hospitality of both the UFRJ team, lead by Professor Fernando Marroquim, and of the organizers, in particular Professor Adilson Jose da Silva, at this most stimulating and enjoyable XXVENFPC Brazilian National Meeting on Particles and Fields, in the beautiful settings of Caxambu. 